\title{
On a Schrödinger-Poisson system with singularity and critical nonlinearities
}

\author{
Zhipeng Cai' , Chunyu Lei' and Changmu Chu ${ }^{1 *}$
}

\section{"Correspondence:}

gzmychuchangmu@sina.com

'School of Data Science and

Information Engineering, Guizhou

Minzu University, Guiyang, People's

Republic of China

\begin{abstract}
In this paper, we study the Schrödinger-Poisson system with singularity and critical growth terms. By means of variational methods with an appropriate truncation argument, the existence and multiplicity of positive solutions are obtained.
\end{abstract}

MSC: 35A15; 35J10; 35J50; 35J75

Keywords: Schrödinger-Poisson system; Critical nonlinearity; Singularity; Positive solutions

\section{Introduction and main result}

In this article, we study the existence and multiplicity of positive solutions to the following Schrödinger-Poisson system:

$$
\begin{cases}-\Delta u-\phi u=u^{5}+\frac{\lambda}{u^{\gamma}}, & \text { in } \Omega, \\ -\Delta \phi=u^{2}, & \text { in } \Omega, \\ u=\phi=0, & \text { on } \partial \Omega,\end{cases}
$$

where $\Omega \subset \mathbb{R}^{3}$ is a smooth bounded domain, $0<\gamma<1, \lambda>0$ is a real parameter. It is well known that system (1.1) is related to the following system:

$$
\begin{cases}-\Delta u+V u+\phi u=f(x, u), & \text { in } \mathbb{R}^{3}, \\ -\Delta \phi=u^{2}, & \text { in } \mathbb{R}^{3},\end{cases}
$$

which was firstly introduced by Benci and Fortunato in [1]. It described the quantum mechanics models and semiconductor theory. We can learn more details about physical background from [2,3] and the references therein. System (1.2) has been extensively studied, focusing on the existence of positive solutions, multiplicity of solutions, ground state solutions, sign-changing solutions, radial solutions, by using the variational methods and critical point theory under various assumptions of potential $V$ and nonlocal term $f$, see for example [4-17] and the references therein.

In addition, existence and multiplicity of the Schrödinger-Poisson problem in a bounded domain has been paid attention to by many authors, we can see [18-24]. More precisely,

(c) The Author(s) 2020. This article is licensed under a Creative Commons Attribution 4.0 International License, which permits use, sharing, adaptation, distribution and reproduction in any medium or format, as long as you give appropriate credit to the original author(s) and the source, provide a link to the Creative Commons licence, and indicate if changes were made. The images or other third party material in this article are included in the article's Creative Commons licence, unless indicated otherwise in a credit line to the material. If material is not included in the article's Creative Commons licence and your intended use is not permitted by statutory regulation or exceeds the permitted use, you will need to obtain permission directly from the copyright holder. To view a copy of this licence, visit http://creativecommons.org/licenses/by/4.0/. 
Fan [21] considered the following system:

$$
\begin{cases}-\Delta u+l(x) \phi u=f_{\lambda}|u|^{q-1} u+g(x) u^{5}, & \text { in } \Omega, \\ -\Delta \phi=l(x) u^{2}, & \text { in } \Omega, \\ u=\phi=0, & \text { on } \partial \Omega,\end{cases}
$$

where $\Omega \subset \mathbb{R}^{3}$ is a bounded domain with smooth boundary, $1<q<2$, and the functions $l(x), f_{\lambda}$, and $g(x)$ satisfy some assumptions, the author proved multiple positive solutions with the help of Nehari manifold and Ljusternik-Schnirelmann category theory.

Zhang in [22] considered the system involving singularity on bounded domain as follows:

$$
\begin{cases}-\Delta u+\eta \phi u=\lambda u^{-\gamma}, & \text { in } \Omega, \\ -\Delta \phi=u^{2}, & \text { in } \Omega, \\ u>0, & \text { in } \Omega, \\ u=\phi=0, & \text { on } \partial \Omega .\end{cases}
$$

For $\eta=1$ and $\lambda>0$, the author obtained the existence and uniqueness of positive solution of system (1.3) by using variational method; for $\eta=-1$ and $\lambda>0$ small enough, the author also considered the existence and multiplicity of positive solutions via Nehari manifold. For the case that replaced with concave-convex nonlinearities and critical growth terms of system (1.3), the authors in [23] got two positive solutions by using the variational method and the concentration-compactness principle when $\lambda$ is small enough.

Recently, Zheng [24] studied the following Schrödinger-Poisson system:

$$
\begin{cases}-\Delta u-\phi u=u^{5}+\lambda u^{q-1}, & \text { in } \Omega, \\ -\Delta \phi=u^{2}, & \text { in } \Omega, \\ u=\phi=0, & \text { on } \partial \Omega,\end{cases}
$$

where $2<q<6, \lambda>0$ is a parameter, the authors obtained one positive ground state solution with the mountain pass theorem and the concentration compactness principle.

As far as we know, there have been no works concerning the existence for system (1.1) up to now. Motivated by the above papers, we study the Schrödinger-Poisson system involving critical and weak singular nonlinearities. Compared with the above mentioned papers, our system has a special point, which makes it difficult to estimate the critical value level. In order to overcome the difficulty, we shall give a special estimate so that two positive solutions of the system can be found by applying the variational method.

Now, our main result is as follows:

Theorem 1.1 Assume that $\gamma \in(0,1)$, then there exists $\lambda_{*}>0$ such that, for any $\lambda \in\left(0, \lambda_{*}\right)$, system (1.1) has at least two pairs of different positive solutions.

\section{Preliminaries}

In this section, we give the variational setting for system (1.1) and use the following notations: 
$H_{0}^{1}(\Omega)$ is the usual Sobolev space with the norm $\|u\|=\left(\int_{\Omega}|\nabla u|^{2} d x\right)^{1 / 2}$, and the norm in $L^{p}(\Omega)$ is represented by $|u|_{p}=\left(\int_{\Omega}|u|^{p} d x\right)^{1 / p}$. We denote by $B_{r}$ (respectively, $\partial B_{r}$ ) the closed ball (respectively, the sphere) of center zero and radius $r . u_{n}^{+}(x)=\max \left\{u_{n}, 0\right\}, u_{n}^{-}(x)=$ $\max \left\{-u_{n}, 0\right\} . C_{1}, C_{2}, C_{3}, \ldots$ denote various positive constants, which may vary from line to line. $S$ is the best Sobolev constant, namely

$$
S=\inf _{u \in H_{0}^{1}\{\Omega\} \backslash\{0\}} \frac{\int_{\Omega}|\nabla u|^{2} d x}{\left(\int_{\Omega}|u|^{6} d x\right)^{\frac{1}{3}}} .
$$

By using the Lax-Milgram theorem, for each $u \in H_{0}^{1}(\Omega)$, there exists a unique solution $\phi_{u}$ which satisfies the second equation of system (1.1). We substitute $\phi_{u}$ to the first equation of system (1.1), we can rewrite system (1.1) as follows:

$$
\begin{cases}-\Delta u-\phi_{u} u=u^{5}+\lambda u^{-\gamma}, & \text { in } \Omega, \\ u=0, & \text { on } \partial \Omega .\end{cases}
$$

Now we define the energy functional $I_{\lambda}$ on $u \in H_{0}^{1}(\Omega)$ by

$$
I_{\lambda}(u)=\frac{1}{2}\|u\|^{2}-\frac{1}{4} \int_{\Omega} \phi_{u}\left(u^{+}\right)^{2} d x-\frac{1}{6} \int_{\Omega}\left(u^{+}\right)^{6} d x-\frac{\lambda}{1-\gamma} \int_{\Omega}\left(u^{+}\right)^{1-\gamma} d x .
$$

If a function $u \in H_{0}^{1}(\Omega)$ satisfies

$$
\int_{\Omega}(\nabla u, \nabla v) d x-\int_{\Omega} \phi_{u}\left(u^{+}\right) v d x-\int_{\Omega}\left(u^{+}\right)^{5} v d x-\lambda \int_{\Omega}\left(u^{+}\right)^{-\gamma} v d x=0
$$

for $v \in H_{0}^{1}(\Omega)$, then we say $u$ is a weak solution of $(2.2)$ and $\left(u, \phi_{u}\right)$ is a pair solution of system (1.1).

Because of the singular nonlinearity $u^{-\gamma}$, the functional $I_{\lambda}$ on $H_{0}^{1}(\Omega)$ is not differentiable. Therefore, we cannot apply directly the usual critical point theory to solve this problem. However, we can find two positive solutions by an approximation approach. That is, for $\alpha>0$, we consider the following perturbation problem:

$$
\begin{cases}-\Delta u-\phi_{u} u=\left(u^{+}\right)^{5}+\frac{\lambda}{\left(u^{+}+\alpha\right)^{\gamma}}, & \text { in } \Omega, \\ u=0, & \text { on } \partial \Omega .\end{cases}
$$

The solution of problem (2.3) corresponds to critical point of the $C^{1}$-functional on $H_{0}^{1}(\Omega)$ by

$$
\begin{aligned}
I_{\lambda, \alpha}(u)= & \frac{1}{2}\|u\|^{2}-\frac{1}{4} \int_{\Omega} \phi_{u}\left(u^{+}\right)^{2} d x-\frac{1}{6} \int_{\Omega}\left(u^{+}\right)^{6} d x \\
& -\frac{\lambda}{1-\gamma} \int_{\Omega}\left(u^{+}+\alpha\right)^{1-\gamma}-\alpha^{1-\gamma} d x .
\end{aligned}
$$

Moreover, if a function $u \in H_{0}^{1}(\Omega)$, and for $v \in H_{0}^{1}(\Omega)$, then $\left(u, \phi_{u}\right)$ is a pair solution of problem (2.3) satisfying

$$
\int_{\Omega}(\nabla u, \nabla v) d x-\int_{\Omega} \phi_{u}\left(u^{+}\right) v d x-\int_{\Omega}\left(u^{+}\right)^{5} v d x-\lambda \int_{\Omega} \frac{v}{\left(u^{+}+\alpha\right)^{\gamma}} d x=0 .
$$




\section{Existence of positive solution for problem (2.3)}

Before proving Theorem 1.1, we recall the following lemma (see [1, 22]).

Lemma 3.1 For every $u \in H_{0}^{1}(\Omega)$, there exists a unique solution $\phi_{u} \in H_{0}^{1}(\Omega)$ of

$$
\begin{cases}-\Delta \phi=u^{2}, & \text { in } \Omega, \\ \phi=0, & \text { on } \partial \Omega\end{cases}
$$

and

(1) $\left\|\phi_{u}\right\|^{2}=\int_{\Omega} \phi_{u} u^{2} d x$;

(2) $\phi_{u} \geq 0$; moreover, $\phi_{u}>0$ when $u \neq 0$;

(3) For each $t \neq 0, \phi_{t u}=t^{2} \phi_{u}$;

(4) $\int_{\Omega} \phi_{u} u^{2} d x=\int_{\Omega}\left|\nabla \phi_{u}\right|^{2} d x \leq S^{-1}|u|_{4}^{4}|\Omega|^{2 / 3} \leq S^{-1}|u|_{12 / 5}^{4} \leq S^{-3}\|u\|^{4}|\Omega|$;

(5) Assume that $u_{n} \rightarrow u$ in $H_{0}^{1}(\Omega)$, then $\phi_{u_{n}} \rightarrow \phi_{u}$ in $H_{0}^{1}(\Omega)$ and

$$
\int_{\Omega} \phi_{u_{n}} u_{n} v d x \rightarrow \int_{\Omega} \phi_{u} u v d x, \quad \forall v \in H_{0}^{1}(\Omega)
$$

(6) Set $\mathcal{F}(u)=\int_{\Omega} \phi_{u} u^{2} d x$, then $\mathcal{F}(u): H_{0}^{1}(\Omega) \rightarrow H_{0}^{1}(\Omega)$ is $C^{1}$ and

$$
\left\langle\mathcal{F}^{\prime}(u), v\right\rangle=4 \int_{\Omega} \phi_{u} u v d x, \quad \forall v \in H_{0}^{1}(\Omega)
$$

Lemma 3.2 There exist $\Lambda_{0}, \rho>0$ such that, for every $\lambda \in\left(0, \Lambda_{0}\right)$, we have

$$
I_{\lambda, \alpha}(u) \geq \kappa \quad \text { for } u \in \overline{\partial B_{\rho}} \quad \text { and } \quad I_{\lambda, \alpha}(u)<0 \quad \text { for } u \in \overline{B_{\rho}} \text {. }
$$

Proof of Lemma 3.2 According to Hölder's inequality and (2.1), we have

$$
\int_{\Omega}\left(u^{+}\right)^{1-\gamma} d x \leq \int_{\Omega}|u|^{1-\gamma} d x \leq|u|_{6}^{1-\gamma}|\Omega|^{\frac{5+\gamma}{6}} \leq|\Omega|^{\frac{5+\gamma}{6}} S^{-\frac{1-\gamma}{2}}\|u\|^{1-\gamma}
$$

Note the subadditivity of $t^{1-\gamma}$, namely

$$
\left(u^{+}+\alpha\right)^{1-\gamma}-\alpha^{1-\gamma} \leq\left(u^{+}\right)^{1-\gamma}, \quad \forall u \in H_{0}^{1}(\Omega) .
$$

It follows from (2.1), (3.2), and (3.3) that

$$
\begin{aligned}
I_{\lambda, \alpha}(u) & =\frac{1}{2}\|u\|^{2}-\frac{1}{4} \int_{\Omega} \phi_{u}\left(u^{+}\right)^{2} d x-\frac{1}{6} \int_{\Omega}\left(u^{+}\right)^{6} d x-\frac{\lambda}{1-\gamma} \int_{\Omega}\left(u^{+}+\alpha\right)^{1-\gamma}-\alpha^{1-\gamma} d x \\
& \geq \frac{1}{2}\|u\|^{2}-\frac{1}{4} \int_{\Omega} \phi_{u}\left(u^{+}\right)^{2} d x-\frac{1}{6} \int_{\Omega}\left(u^{+}\right)^{6} d x-\frac{\lambda}{1-\gamma} \int_{\Omega}\left(u^{+}\right)^{1-\gamma} \\
& \geq \frac{1}{2}\|u\|^{2}-\frac{|\Omega|}{4} S^{-3}\|u\|^{4}-\frac{1}{6} S^{-3}\|u\|^{6}-\frac{\lambda}{1-\gamma}|\Omega|^{\frac{5+\gamma}{6}} S^{-\frac{1-\gamma}{2}}\|u\|^{1-\gamma} \\
& \geq\|u\|^{1-\gamma}\left(\frac{1}{2}\|u\|^{1+\gamma}-\frac{|\Omega|}{4} S^{-3}\|u\|^{3+\gamma}-\frac{1}{6} S^{-3}\|u\|^{5+\gamma}-\frac{\lambda}{1-\gamma}|\Omega|^{\frac{5+\gamma}{6}} S^{-\frac{1-\gamma}{2}}\right) .
\end{aligned}
$$


Set $g(t)=\frac{1}{2} t^{1+\gamma}-\frac{|\Omega|}{4} S^{-3} t^{3+\gamma}-\frac{1}{6} S^{-3} t^{5+\gamma}$ for $t>0$, then there exists a positive constant

$$
\rho=\left[\frac{-3(3+\gamma)|\Omega|+\sqrt{9(3+\gamma)^{2}|\Omega|^{2}+48\left(5+6 \gamma+\gamma^{2}\right) S^{3}}}{4(5+\gamma)}\right]^{\frac{1}{2}}>0
$$

such that $\max _{t>0} g(t)=g(\rho)>0$. Letting $\Lambda_{0}=\frac{(1-\gamma) S^{\frac{1-\gamma}{2}}}{2|\Omega|^{\frac{5+\gamma}{6}}} g(\rho)$, it follows that there exists a constant $\kappa>0$ such that $\left.I_{\lambda, \alpha}(u)\right|_{S_{\rho}} \geq \kappa$ for every $\lambda \in\left(0, \Lambda_{0}\right)$.

Especially, we define a function $f(x)=x^{1-\gamma}, x \in \Omega$, by using the Lagrange mean value theorem, there exists $\xi>0$ such that

$$
\left(u^{+}+\alpha\right)^{1-\gamma}-\alpha^{1-\gamma}=f^{\prime}(\xi) u^{+}
$$

here $\xi \in\left(\alpha, u^{+}+\alpha\right)$. For every $u \in \overline{B_{\rho}}, u^{+} \neq 0$, we have

$$
\begin{aligned}
\lim _{t \rightarrow 0^{+}} \frac{I_{\lambda, \alpha}(t u)}{t} & =-\frac{\lambda}{1-\gamma} \lim _{t \rightarrow 0^{+}} \frac{1}{t} \int_{\Omega}\left(t u^{+}+\alpha\right)^{1-\gamma}-\alpha^{1-\gamma} d x \\
& =-\lambda \int_{\Omega} \xi^{-\gamma} t u^{+} d x \\
& <0 .
\end{aligned}
$$

For $t$ small enough, we have $I_{\lambda, \alpha}(t u)<0$. Hence, there exists $u$ small enough such that $I_{\lambda, \alpha}(u)<0$. Therefore, we deduce that

$$
d=: \inf _{u \in \overline{B_{\rho}}} I_{\lambda, \alpha}(u)<\inf _{u \in \overline{\partial B_{\rho}}} I_{\lambda, \alpha}(u) .
$$

The proof is complete.

Lemma 3.3 Let $0<\alpha<1$, if $\left\{u_{n}\right\} \subset H_{0}^{1}(\Omega)$ is a $(P S)_{c}$ sequence for $I_{\lambda, \alpha}$ with $c<\frac{1}{3} S^{\frac{2}{2}}-D \lambda^{\frac{2}{1+\gamma}}$, where $D=\frac{1+\gamma}{4(1-\gamma)}\left(\frac{3+\gamma}{2}|\Omega|^{\frac{5+\gamma}{6}} S^{-\frac{1-\gamma}{2}}\right)^{\frac{2}{1+\gamma}}$, then there exists $u_{0} \in H_{0}^{1}(\Omega)$ such that $u_{n} \rightarrow u_{0}$ in $H_{0}^{1}(\Omega)$ and $\int_{\Omega} u_{n}^{6} d x \rightarrow \int_{\Omega} u_{0}^{6} d x$.

Proof of Lemma 3.3 Let $\left\{u_{n}\right\} \subset H_{0}^{1}(\Omega)$ be such that

$$
I_{\lambda, \alpha}\left(u_{n}\right) \rightarrow c, \quad I_{\lambda, \alpha}^{\prime}\left(u_{n}\right) \rightarrow 0
$$

Now, we claim that $\left\{u_{n}\right\}$ is bounded in $H_{0}^{1}(\Omega)$. Otherwise, we assume that $\left\|u_{n}\right\| \rightarrow \infty$, as $n \rightarrow \infty$. It follows from (3.2), (3.3), and (3.4) that

$$
\begin{aligned}
1+c+o(1)\left\|u_{n}\right\| & =I_{\lambda, \alpha}\left(u_{n}\right)-\frac{1}{4}\left\langle I_{\lambda, \alpha}^{\prime}\left(u_{n}\right), u_{n}\right\rangle \\
& \geq \frac{1}{4}\left\|u_{n}\right\|^{2}+\frac{1}{12} \int_{\Omega}\left(u^{+}\right)^{6} d x-\frac{\lambda}{1-\gamma} \int_{\Omega}\left[\left(u_{n}^{+}+\alpha\right)^{1-\gamma}-\alpha^{1-\gamma}\right] d x \\
& \geq \frac{1}{4}\left\|u_{n}\right\|^{2}-\frac{\lambda}{1-\gamma} \int_{\Omega}\left(u_{n}^{+}\right)^{1-\gamma} d x \\
& \geq \frac{1}{4}\left\|u_{n}\right\|^{2}-\frac{\lambda}{1-\gamma}|\Omega|^{\frac{5+\gamma}{6}} S^{-\frac{1-\gamma}{2}}\left\|u_{n}^{+}\right\|^{1-\gamma} .
\end{aligned}
$$


Since $0<\gamma<1$, the last inequality above is impossible, which implies that $\left\{u_{n}\right\}$ is bounded in $H_{0}^{1}(\Omega)$. So there exists $\tau \in L^{1}(\Omega)$ for all $n$ such that $\left|u_{n}(x)\right| \leq \tau(x)$ a.e. in $\Omega$. And there exists a subsequence, still denoted by $\left\{u_{n}\right\}$. We assume that there exists $u_{0} \in H_{0}^{1}(\Omega)$ such that

$$
\left\{\begin{array}{l}
u_{n} \rightarrow u_{0}, \quad \text { weakly in } H_{0}^{1}(\Omega), \\
u_{n} \rightarrow u_{0}, \quad \text { strongly in } L^{p}(\Omega)(1 \leq p<6), \\
u_{n}(x) \rightarrow u_{0}(x), \quad \text { a.e. in } \Omega .
\end{array}\right.
$$

Note the given condition $\alpha>0$, we can easily get $\frac{\left|u_{0}\right|}{\left(u_{0}^{+}+\alpha\right)^{\gamma}} \leq \frac{\left|u_{0}\right|}{\alpha^{\gamma}}$. Then, by the dominated convergence theorem and (3.5), we have

$$
\lim _{n \rightarrow \infty} \int_{\Omega}\left(u_{n}^{+}+\alpha\right)^{-\gamma} u_{0} d x=\int_{\Omega}\left(u_{0}^{+}+\alpha\right)^{-\gamma} u_{0} d x
$$

Moreover, we have $\left|\frac{u_{n}}{\left(u_{n}^{+}+\alpha\right)^{\gamma}}\right| \leq \frac{\tau}{\alpha^{\gamma}}$, by the dominated convergence theorem, we also have

$$
\lim _{n \rightarrow \infty} \int_{\Omega}\left(u_{n}^{+}+\alpha\right)^{-\gamma} u_{n} d x=\int_{\Omega}\left(u_{0}^{+}+\alpha\right)^{-\gamma} u_{0} d x
$$

Now, set $w_{n}=u_{n}-u_{0}$, then $\left\|w_{n}\right\| \rightarrow 0$ as $n \rightarrow \infty$. Otherwise, there exists a subsequence, still denoted by $w_{n}$, such that

$$
\lim _{n \rightarrow \infty}\left\|w_{n}\right\|=l>0
$$

Note that $\lim _{n \rightarrow \infty}\left\langle I_{\lambda, \alpha}^{\prime}\left(u_{n}\right), u_{0}\right\rangle=0$ and (3.6), we deduce

$$
\left\|u_{0}\right\|^{2}-\int_{\Omega} \phi_{u_{0}}\left(u_{0}^{+}\right)^{2} d x-\int_{\mathbb{R}^{3}}\left(u_{0}^{+}\right)^{6} d x-\lambda \int_{\Omega}\left(u_{0}^{+}+\alpha\right)^{-\gamma} u_{0} d x=0 .
$$

Using the Brézis-Lieb lemma [25], we have

$$
\left\{\begin{array}{l}
\left\|u_{n}\right\|^{2}=\left\|w_{n}\right\|^{2}+\left\|u_{0}\right\|^{2}+o(1), \\
\int_{\Omega}\left(u_{n}^{+}\right)^{6} d x=\int_{\Omega}\left(w_{n}^{+}\right)^{6} d x+\int_{\Omega}\left(u_{0}^{+}\right)^{6} d x+o(1) .
\end{array}\right.
$$

It follows from (3.4), (3.7), and (3.9) that

$$
\begin{aligned}
o(1)= & \left\|w_{n}\right\|^{2}+\left\|u_{0}\right\|^{2}-\int_{\Omega} \phi_{u_{0}}\left(u_{0}^{+}\right)^{2} d x \\
& -\int_{\Omega}\left(w_{n}^{+}\right)^{6} d x-\int_{\Omega}\left(u_{0}^{+}\right)^{6} d x-\lambda \int_{\Omega}\left(u_{0}^{+}+\alpha\right)^{-\gamma} u_{0} d x
\end{aligned}
$$

Therefore, (3.8) and (3.10) lead to

$$
\left\|w_{n}\right\|^{2}-\int_{\Omega}\left(w_{n}^{+}\right)^{6} d x=o(1)
$$

Since also $\int_{\Omega}\left(w_{n}^{+}\right)^{6} d x \leq \int_{\Omega}\left|w_{n}\right|^{6} d x$, then, according to (2.1), (3.11) implies that

$$
l^{2} \geq S^{\frac{3}{2}} .
$$


From (3.2) and using the Young inequality, we have

$$
\begin{aligned}
I_{\lambda, \alpha}\left(u_{0}\right)= & \frac{1}{2}\left\|u_{0}\right\|^{2}-\frac{1}{4} \int_{\Omega} \phi_{u_{0}}\left(u_{0}^{+}\right)^{2} d x-\frac{1}{6} \int_{\Omega} u_{0}^{6} d x \\
& -\frac{\lambda}{1-\gamma} \int_{\Omega}\left[\left(u_{0}^{+}+\alpha\right)^{1-\gamma}-\alpha^{1-\gamma}\right] d x \\
\geq & \frac{1}{2}\left\|u_{0}\right\|^{2}-\frac{1}{4} \int_{\Omega} \phi_{u_{0}}\left(u_{0}^{+}\right)^{2} d x-\frac{1}{6} \int_{\Omega} u_{0}^{6} d x-\frac{\lambda}{1-\gamma} \int_{\Omega}\left(u_{0}^{+}\right)^{1-\gamma} d x \\
= & \frac{1}{4}\left\|u_{0}\right\|^{2}+\frac{1}{12} \int_{\Omega} u_{0}^{6} d x-\lambda\left(\frac{1}{1-\gamma}-\frac{1}{4}\right) \int_{\Omega}\left(u_{0}^{+}\right)^{1-\gamma} d x \\
\geq & \frac{1}{4}\left\|u_{0}\right\|^{2}-\lambda\left(\frac{1}{1-\gamma}-\frac{1}{4}\right)|\Omega|^{\frac{5+\gamma}{6}} S^{-\frac{1-\gamma}{2}}\left\|u_{0}\right\|^{1-\gamma} \\
\geq & -D \lambda \frac{2}{1+\gamma},
\end{aligned}
$$

where $D=\frac{1+\gamma}{4(1-\gamma)}\left(\frac{3+\gamma}{2}|\Omega|^{\frac{5+\gamma}{6}} S^{-\frac{1-\gamma}{2}}\right)^{\frac{2}{1+\gamma}}$. Combining (3.10) with (3.11), we also have

$$
\begin{aligned}
I_{\lambda, \alpha}\left(u_{0}\right) & =I_{\lambda, \alpha}\left(u_{n}\right)-\frac{1}{2}\left\|w_{n}\right\|^{2}+\frac{1}{6} \int_{\Omega}\left|w_{n}\right|^{6} d x+o(1) \\
& =c-\frac{1}{3}\left\|w_{n}\right\|^{2}+o(1) \\
& <c-\frac{1}{3} S^{\frac{3}{2}} \\
& =\frac{1}{3} S^{\frac{2}{2}}-D \lambda^{\frac{2}{1+\gamma}}-\frac{1}{3} S^{\frac{3}{2}} \\
& =-D \lambda^{\frac{2}{1+\gamma}} .
\end{aligned}
$$

It is obvious that the above two inequalities are impossibility. Thus, we get $l=0$, which yields $u_{n} \rightarrow u_{0}$ in $H_{0}^{1}(\Omega)$. By (3.11), we get

$$
0 \leq \int_{\Omega} u_{n}^{6} d x-\int_{\Omega} u_{0}^{6} d x=\int_{\Omega} w_{n}^{6} d x+o(1)=\left\|w_{n}\right\|^{2} \rightarrow 0
$$

which implies that $\int_{\Omega} u_{n}^{6} d x \rightarrow \int_{\Omega} u_{0}^{6} d x$ as $n \rightarrow \infty$. The proof is complete.

Note that $0<\alpha<1$, we can get

$$
\begin{aligned}
I_{\lambda, \alpha}(u) & =\frac{1}{2}\|u\|^{2}-\frac{1}{4} \int_{\Omega} \phi_{u}\left(u^{+}\right)^{2} d x-\frac{1}{6} \int_{\Omega}\left(u^{+}\right)^{6} d x-\frac{\lambda}{1-\gamma} \int_{\Omega}\left(u^{+}+\alpha\right)^{1-\gamma}-\alpha^{1-\gamma} d x \\
& \leq \frac{1}{2}\|u\|^{2}-\frac{1}{6} \int_{\Omega}\left(u^{+}\right)^{6} d x-\frac{\lambda}{1-\gamma} \int_{\Omega}\left(u^{+}+\alpha\right)^{1-\gamma} d x+\frac{\lambda}{1-\gamma} \int_{\Omega} \alpha^{1-\gamma} d x \\
& \leq \frac{1}{2}\|u\|^{2}-\frac{1}{6} \int_{\Omega}\left(u^{+}\right)^{6} d x-\frac{\lambda}{1-\gamma} \int_{\Omega}\left(u^{+}\right)^{1-\gamma} d x+\frac{\lambda}{1-\gamma}|\Omega| .
\end{aligned}
$$

Now, we define a new functional $J_{\lambda}(u): H_{0}^{1}(\Omega) \rightarrow \mathbb{R}$ as follows:

$$
J_{\lambda}(u)=\frac{1}{2}\|u\|^{2}-\frac{1}{6} \int_{\Omega}\left(u^{+}\right)^{6} d x-\frac{\lambda}{1-\gamma} \int_{\Omega}\left(u^{+}\right)^{1-\gamma} d x .
$$


Consequently, we consider the following problem:

$$
\begin{cases}-\Delta u=u^{5}+\frac{\lambda}{u^{\gamma}}, & \text { in } \Omega, \\ u=0, & \text { on } \Omega .\end{cases}
$$

And we find that the weak solutions of problem (3.14) correspond to the critical points of the functional $J_{\lambda}$.

Remark 3.4 There exists $\rho, \Lambda_{0}>0$ (given by Lemma 3.2 such that problem (3.14) has a positive solution $v_{0} \in \overline{B_{\rho}}$ with $J_{\lambda}\left(v_{0}\right)<0$ and $\left.J_{\lambda}\right|_{\overline{\partial B_{\rho}}}>0$ for every $\lambda \in\left(0, \Lambda_{0}\right)$. In fact, from (3.13), we have

$$
\begin{aligned}
J_{\lambda}(u) & =\frac{1}{2}\|u\|^{2}-\frac{1}{6} \int_{\Omega}\left(u^{+}\right)^{6} d x-\frac{\lambda}{1-\gamma} \int_{\Omega}\left(u^{+}\right)^{1-\gamma} \\
& \geq \frac{1}{2}\|u\|^{2}-\frac{1}{6} S^{-3}\|u\|^{6}-\frac{\lambda}{1-\gamma}|\Omega|^{\frac{5+\gamma}{6}} S^{-\frac{1-\gamma}{2}}\|u\|^{1-\gamma} \\
& \geq\|u\|^{1-\gamma}\left(\frac{1}{2}\|u\|^{1+\gamma}-\frac{1}{6} S^{-3}\|u\|^{5+\gamma}-\frac{\lambda}{1-\gamma}|\Omega|^{\frac{5+\gamma}{6}} S^{-\frac{1-\gamma}{2}}\right) .
\end{aligned}
$$

By Lemma 3.2, when $\|u\|=\rho$, we have

$$
\frac{1}{2} \rho^{1+\gamma}-\frac{|\Omega|}{4} S^{-3} \rho^{3+\gamma}-\frac{1}{6} S^{-3} \rho^{5+\gamma}-\frac{\lambda}{1-\gamma}|\Omega|^{\frac{5+\gamma}{6}} S^{-\frac{1-\gamma}{2}}>0
$$

for every $\lambda \in\left(0, \Lambda_{0}\right)$. Then we deduce that $\left.J_{\lambda}\right|_{\overline{\partial B_{\rho}}}>0$ for $\lambda \in\left(0, \Lambda_{0}\right)$. Similar to Lemma 3.2, we get $v_{0} \in \overline{B_{\rho}}$ and $J_{\lambda}\left(v_{0}\right)<0$ for every $\lambda \in\left(0, \Lambda_{0}\right)$. Moreover, there exist two constants $m, M>0$ such that $m<v_{0}(x)<M$.

As usual, we consider the following function:

$$
U_{\varepsilon}(x)=\frac{\left(3 \varepsilon^{2}\right)^{\frac{1}{2}}}{\left(\varepsilon^{2}+|x|^{2}\right)^{\frac{1}{2}}}
$$

where $\varepsilon$ is a positive constant. Moreover, we know that $U_{\varepsilon}$ is a positive solution of problem $-\Delta u=|u|^{4} u$ in $\mathbb{R}^{3}$ and $\int_{\Omega}\left|\nabla U_{\varepsilon}\right|^{2} d x=\int_{\Omega}\left|U_{\varepsilon}\right|^{6}+S^{\frac{3}{2}}$. Let $\zeta$ be a smooth cut-off function $\zeta \in C_{0}^{\infty}(\Omega)$ such that $0 \leq \zeta(x) \leq 1$ in $\Omega$. $\zeta(x)=1$ near $x=0$ and it is radially symmetric. Set $v_{\varepsilon}(x)=\zeta(x) U(x)$. Then we have the following.

Lemma 3.5 Assume $0<\gamma<1$, there holds

$$
\sup _{t \geq 0} I_{\lambda, \alpha}\left(v_{0}+t v_{\varepsilon}\right)<\frac{1}{3} S^{\frac{3}{2}}-D \lambda^{\frac{2}{1+\gamma}}
$$

Proof of Lemma 3.5 From [26], one has

$$
\int_{\Omega}\left|\nabla v_{\varepsilon}\right|^{2} d x=S^{\frac{3}{2}}+O(\varepsilon), \quad \int_{\Omega}\left|v_{\varepsilon}(x)\right|^{6} d x=S^{\frac{3}{2}}+O\left(\varepsilon^{3}\right)
$$


It is well known that the following inequality

$$
(a+b)^{6} \geq a^{6}+b^{6}+6 a^{5} b+6 a b^{5}
$$

holds true for each $a, b \geq 0$. With no loss of generality, for $a \geq m$ and $b \geq 0$, we can get that

$$
(a+b)^{1-\gamma}-a^{1-\gamma} \geq 0
$$

Since $v_{0}$ is a positive solution of problem (3.14), then there holds

$$
\begin{aligned}
& J_{\lambda}\left(v_{0}+t v_{\varepsilon}\right) \\
&=\frac{1}{2}\left\|v_{0}+t v_{\varepsilon}\right\|^{2}-\frac{1}{6} \int_{\Omega}\left(v_{0}+t v_{\varepsilon}\right)^{6} d x-\frac{\lambda}{1-\gamma} \int_{\Omega}\left(v_{0}+t v_{\varepsilon}\right)^{1-\gamma} d x \\
&=I_{\lambda}\left(v_{0}\right)+\frac{t^{2}}{2}\left\|v_{\varepsilon}\right\|^{2}+t \int_{\Omega}\left[\left(\nabla v_{0}, \nabla v_{\varepsilon}\right)-v_{0}^{5} v_{\varepsilon}-\lambda v_{0}^{-\gamma} v_{\varepsilon}\right] d x-\frac{1}{6} \int_{\Omega}\left[\left|v_{0}+t v_{\varepsilon}\right|^{6}\right. \\
&\left.\quad-v_{0}^{6}-6 v_{0}^{5} t v_{\varepsilon}\right] d x-\frac{\lambda}{1-\gamma} \int_{\Omega}\left[\left|v_{0}+t v_{\varepsilon}\right|^{1-\gamma}-v_{0}^{1-\gamma}-(1-\gamma) v_{0}^{-\gamma} t u_{\varepsilon}\right] d x \\
& \leq I_{\lambda}\left(v_{0}\right)+\frac{t^{2}}{2}\left\|v_{\varepsilon}\right\|^{2}+t \int_{\Omega}\left[\left(\nabla v_{0}, \nabla v_{\varepsilon}\right)-v_{0}^{5} v_{\varepsilon}-\lambda v_{0}^{-\gamma} v_{\varepsilon}\right] d x-\frac{1}{6} \int_{\Omega}\left[\left|v_{0}+t v_{\varepsilon}\right|^{6}\right. \\
&\left.\quad-v_{0}^{6}-6 v_{0}^{5} t v_{\varepsilon}\right] d x+\lambda \int_{\Omega} v_{0}^{-\gamma} t v_{\varepsilon} d x \\
& \leq \leq \frac{t^{2}}{2}\left\|v_{\varepsilon}\right\|^{2}-\frac{t^{6}}{6} \int_{\Omega}\left|v_{\varepsilon}\right|^{6} d x-t^{5} \int_{\Omega} v_{0}\left|v_{\varepsilon}\right|^{5} d x+\lambda \int_{\Omega} v_{0}^{-\gamma} t v_{\varepsilon} d x \\
& \leq \frac{t^{2}}{2}\left\|v_{\varepsilon}\right\|^{2}-\frac{t^{6}}{6} \int_{\Omega}\left|v_{\varepsilon}\right|^{6} d x-m t^{5} \int_{\Omega}\left|v_{\varepsilon}\right|^{5} d x+M^{-\gamma} \lambda t \int_{\Omega} v_{\varepsilon} d x
\end{aligned}
$$

Let

$$
h(t)=\frac{t^{2}}{2}\left\|v_{\varepsilon}\right\|^{2}-\frac{t^{6}}{6} \int_{\Omega}\left|v_{\varepsilon}\right|^{6} d x-m t^{5} \int_{\Omega}\left|v_{\varepsilon}\right|^{5} d x+M^{-\gamma} \lambda t \int_{\Omega} v_{\varepsilon} d x
$$

Similar to paper [27], we can find that there exist $t_{\varepsilon}$ and positive constants $t_{1}, t_{2}$ (independent of $\varepsilon, \lambda)$ such that $\sup _{t \geq 0} h(t)=h\left(t_{\varepsilon}\right)$ and

$$
0<t_{1} \leq t_{\varepsilon} \leq t_{2}<\infty
$$

Indeed, since $\lim _{t \rightarrow 0} h(t)=0, \lim _{t \rightarrow+\infty} h(t)=-\infty$, there exists $t_{\varepsilon}$ such that

$$
h\left(t_{\varepsilon}\right)=\sup _{t \geq 0} h(t), \quad \text { and }\left.\quad h^{\prime}(t)\right|_{t=t_{\varepsilon}}=0 .
$$

Note that $\int_{\Omega}\left|v_{\varepsilon}(x)\right|^{5} d x=C_{1} \varepsilon^{\frac{1}{2}}$ and $\int_{\Omega}\left|v_{\varepsilon}(x)\right| d x=C_{2} \varepsilon^{\frac{1}{2}}$, one has

$$
\begin{aligned}
\sup _{t \geq 0} J_{\lambda}\left(v_{0}+t v_{\varepsilon}\right) & \leq \sup _{t \geq 0} h(t)=h\left(t_{\varepsilon}\right) \\
& \leq \sup _{t \geq 0}\left\{\frac{t^{2}}{2}\left\|u_{\varepsilon}\right\|^{2}-\frac{t^{6}}{6} \int_{\Omega}\left|u_{\varepsilon}\right|^{6} d x\right\}-m t_{1}^{5} \int_{\Omega}\left|v_{\varepsilon}\right|^{5} d x
\end{aligned}
$$




$$
\begin{aligned}
& +M^{-\gamma} \lambda t_{2} \int_{\Omega} v_{\varepsilon} d x \\
\leq & \sup _{t \geq 0}\left\{\frac{t^{2}}{2}\left\|u_{\varepsilon}\right\|^{2}-\frac{t^{6}}{6} \int_{\Omega}\left|u_{\varepsilon}\right|^{6} d x\right\}-C_{3} \varepsilon^{\frac{1}{2}}+\lambda C_{4} \varepsilon^{\frac{1}{2}} \\
\leq & \sup _{t \geq 0}\left\{\frac{t^{2}}{2} S^{\frac{3}{2}}-\frac{t^{6}}{6} S^{\frac{3}{2}}\right\}+C_{5} \varepsilon-C_{3} \varepsilon^{\frac{1}{2}}+\lambda C_{4} \varepsilon^{\frac{1}{2}} \\
\leq & \frac{1}{3} S^{\frac{3}{2}}+C_{5} \varepsilon-C_{3} \varepsilon^{\frac{1}{2}}+\lambda C_{4} \varepsilon^{\frac{1}{2}} .
\end{aligned}
$$

From (3.12), we get the following estimate:

$$
\begin{aligned}
\sup _{t \geq 0} I_{\lambda, \alpha}\left(v_{0}+t v_{\varepsilon}\right) & \leq \sup _{t \geq 0} J_{\lambda}\left(v_{0}+t v_{\varepsilon}\right)+\frac{\lambda}{1-\gamma}|\Omega| \\
& \leq \frac{1}{3} S^{\frac{3}{2}}+C_{5} \varepsilon-C_{3} \varepsilon^{\frac{1}{2}}+\lambda C_{4} \varepsilon^{\frac{1}{2}}+C_{6} \lambda \\
& \leq \frac{1}{3} S^{\frac{3}{2}}+C_{5} \varepsilon+C_{7} \lambda-C_{3} \varepsilon^{\frac{1}{2}} .
\end{aligned}
$$

Let $\varepsilon=\lambda^{\frac{2}{1+\gamma}}$, and for $\frac{2}{1+\gamma}>1$, there holds

$$
\begin{aligned}
C_{5} \varepsilon+C_{7} \lambda-C_{3} \varepsilon^{\frac{1}{2}} & =C_{5} \lambda^{\frac{2}{1+\gamma}}+C_{7} \lambda-C_{3} \lambda^{\frac{1}{1+\gamma}} \\
& \leq \lambda^{\frac{2}{1+\gamma}}\left(C_{8} \lambda^{\frac{\gamma-1}{1+\gamma}}-C_{3} \lambda^{\frac{-1}{1+\gamma}}\right) \\
& =\lambda^{\frac{2}{1+\gamma}}\left(\frac{C_{8}}{\lambda^{\frac{1-\gamma}{1+\gamma}}}-\frac{C_{3}}{\lambda^{1+\gamma}}\right) .
\end{aligned}
$$

As $0<\gamma<1$, we have $\frac{1-\gamma}{1+\gamma}<\frac{1}{1+\gamma}$. Moreover, we get that $\lambda^{\frac{1-\gamma}{1+\gamma}}>\lambda^{\frac{1}{1+\gamma}}$ for every $\lambda \in(0,1)$. Consequently, there exists $\Lambda_{1}>0$ such that $\lambda \leq \Lambda_{1}$, then it is shown that

$$
\frac{C_{8}}{\lambda^{\frac{1-\gamma}{1+\gamma}}}-\frac{C_{3}}{\lambda^{\frac{1}{1+\gamma}}} \leq-D
$$

Thereby, from the above inequality, we conclude that

$$
\sup _{t \geq 0} I_{\lambda, \alpha}\left(v_{0}+t v_{\varepsilon}\right) \leq \frac{1}{3} S^{\frac{3}{2}}-D \lambda^{\frac{2}{1+\gamma}} .
$$

Hence, (3.15) holds true for $\lambda<\min \left\{\Lambda_{0}, \Lambda_{1}\right\}$. The proof is complete.

Theorem 3.6 Assume $0<\alpha<1,0<\gamma<1$, there exists $\lambda_{*}>0$ such that $0<\lambda<\lambda_{*}$, problem (2.3) has at least a positive solution $v_{\alpha} \in H_{0}^{1}(\Omega)$ satisfying $I_{\lambda, \alpha}\left(v_{\alpha}\right)>0$.

Proof of Theorem 3.6 Let $\lambda_{*}=\min \left\{\Lambda_{0}, \Lambda_{1}\right\}$, then Lemmas 3.3 and 3.5 hold for $0<\lambda<\lambda_{*}$. As a matter of fact, according to Remark 3.4, we have $I_{\lambda, \alpha}(0)=0, I_{\lambda, \alpha}\left(v_{0}\right)<0$ and $\left.I_{\lambda, \alpha}\right|_{\overline{B_{\rho}}}>0$. By Lemma 3.5, we can choose $T_{0}>0$ large enough so that $I_{\lambda, \alpha}\left(v_{0}+T_{0} v_{\varepsilon}\right)<0$. Consequently, $I_{\lambda, \alpha}$ satisfies the geometry of the mountain pass lemma [28]. Applying the mountain pass lemma, there exists a sequence $\left\{v_{n}\right\} \subset H_{0}^{1}$ such that

$$
I_{\lambda, \alpha}\left(v_{n}\right) \rightarrow c>0 \quad \text { and } \quad I_{\lambda, \alpha}^{\prime}\left(v_{n}\right) \rightarrow 0, \quad \text { as } n \rightarrow \infty
$$


where

$$
c=\inf _{\gamma \in \Gamma \in[0,1]} \max _{\lambda, \alpha}(\gamma(t))
$$

and

$$
\Gamma=\left\{\gamma \in C\left([0,1], H_{0}^{1}\right): \gamma(0)=u_{0}, \gamma(1)=v_{0}+T_{0} v_{\varepsilon}\right\}
$$

Moreover, by Lemmas 3.2 and 3.5, we get

$$
0<\kappa<c \leq \max _{t \in[0,1]} I_{\lambda, \alpha}\left(v_{0}+T_{0} v_{\varepsilon}\right) \leq \sup _{t \geq 0} I_{\lambda, \alpha}\left(v_{0}+T_{0} v_{\varepsilon}\right)<\frac{1}{3} S^{\frac{3}{2}}-D \lambda^{\frac{2}{1+\gamma}} .
$$

According to Lemma 3.3, we know that $\left\{v_{n}\right\} \subset H_{0}^{1}(\Omega)$ has a convergent subsequence, still denoted by $\left\{v_{n}\right\}$, we may assume that $v_{n} \rightarrow v_{\alpha}$ in $H_{0}^{1}(\Omega)$ as $n \rightarrow \infty$. Hence, from (3.16) and (3.17) we have

$$
I_{\lambda, \alpha}\left(v_{\alpha}\right)=\lim _{n \rightarrow \infty} I_{\lambda, \alpha}\left(v_{n}\right)=c>\kappa>0,
$$

which implies $v_{\alpha} \not \equiv 0$. Furthermore, from the continuity of $I_{\lambda, \alpha}^{\prime}$, we find that $v_{\alpha}$ is a solution of problem (2.3), namely

$$
\int_{\Omega}\left(\nabla v_{\alpha}, \nabla \varphi\right) d x-\int_{\Omega} \phi_{u}\left(v_{\alpha}^{+}\right) \varphi d x-\int_{\Omega}\left(v_{\alpha}^{+}\right)^{5} \varphi d x-\lambda \int_{\Omega} \frac{\varphi}{\left(v_{\alpha}^{+}+\alpha\right)^{\gamma}} d x=0
$$

for all $\varphi \in H_{0}^{1}(\Omega)$. Taking the test function $\varphi=v_{\alpha}^{-}$, we have

$$
-\left\|v_{\alpha}^{-}\right\|^{2}=\lambda \int_{\Omega} \frac{v_{\alpha}^{-}}{\left(v_{\alpha}^{+}+\alpha\right)^{\gamma}} d x \geq 0
$$

we infer that $v_{\alpha}^{-}=0$. Then we deduce that $v_{\alpha} \geq 0$ and $v_{\alpha} \not \equiv 0$. Hence, by the strong maximum principle, we obtain $v_{\alpha}>0$ in $\Omega$ and $v_{\alpha}$ is a positive solution of problem (2.3). The proof is complete.

Theorem 3.7 Assume $0<\alpha<1,0<\gamma<1$, there exists $\lambda_{*}>0$ such that $0<\lambda<\lambda_{*}$, problem (2.3) has at least a positive solution $v_{\alpha} \in H_{0}^{1}(\Omega)$ satisfying $I_{\lambda, \alpha}\left(v_{\alpha}\right)>0$.

Proof of Theorem 3.7 From Lemma 3.2, by applying Ekeland's variational principle in $\overline{B_{\rho}}$, there exists a minimizing sequence $\left\{u_{n}\right\} \subset \overline{B_{\rho}}$ such that

$$
I_{\lambda, \alpha}\left(u_{n}\right) \leq \inf _{u \in \overline{B_{\rho}}} I_{\lambda, \alpha}(u)+\frac{1}{n}, \quad I_{\lambda, \alpha}(v) \geq I_{\lambda, \alpha}\left(u_{n}\right)-\frac{1}{n}\left\|v-u_{n}\right\|, \quad v \in \overline{B_{\rho}} .
$$

Therefore,

$$
I_{\lambda, \alpha}^{\prime}\left(u_{n}\right) \rightarrow 0 \quad \text { and } \quad I_{\lambda, \alpha}\left(u_{n}\right) \rightarrow d .
$$

Since $\left\{u_{n}\right\}$ is bounded and $\overline{B_{\rho}}$ is a closed convex set, there exist $u_{\alpha} \in \overline{B_{\rho}} \subset H_{0}^{1}(\Omega)$ and a subsequence still denoted by $\left\{u_{n}\right\}$ such that $u_{n} \rightarrow u_{\lambda}$ in $H_{0}^{1}(\Omega)$ as $n \rightarrow \infty$. 
Note that $I_{\lambda, \alpha}\left(\left|u_{n}\right|\right)=I_{\lambda, \alpha}\left(u_{n}\right)$, by Lemma 3.3, we can obtain $u_{n} \rightarrow u_{\alpha}$ in $H_{0}^{1}(\Omega)$ and $d=\lim _{n \rightarrow \infty} I_{\lambda, \alpha}\left(u_{n}\right)=I_{\lambda, \alpha}\left(u_{\alpha}\right)<0$, which suggests that $u_{\lambda} \geq 0$ and $u_{\alpha} \neq \equiv 0$. Similar to Theorem 3.6, we obtain $u_{\alpha}>0$ in $\Omega$, then $u_{\alpha}$ is a solution of problem (2.3) with $I_{\lambda, \alpha}\left(u_{\alpha}\right)<0$. The proof is complete.

\section{Existence of positive solutions for system (1.1)}

Proof of Theorem 1.1 Now, we need to prove that system (1.1) has two positive solutions. Let $\left\{v_{\alpha}\right\}$ be a family of positive solutions of problem (2.3), one has

$$
\left\|v_{\alpha}\right\|-\int_{\Omega} \phi_{v_{\alpha}} v_{\alpha}^{2} d x-\int_{\Omega} v_{\alpha}^{6} d x-\lambda \int_{\Omega}\left(v_{\alpha}+\alpha\right)^{-\gamma} v_{\alpha} d x=0
$$

Hence, it follows from (2.1), (3.2), and (4.1) that

$$
\begin{aligned}
\frac{1}{3} S^{\frac{3}{2}}-D \lambda \frac{2}{1+\gamma}> & I_{\lambda, \alpha}\left(v_{\alpha}\right)-\frac{1}{4}\left\langle I_{\lambda, \alpha}^{\prime}\left(v_{\alpha}\right), v_{\alpha}\right\rangle \\
= & \frac{1}{4}\left\|v_{\alpha}\right\|^{2}+\frac{1}{12} \int_{\Omega} v_{\alpha}^{6} d x+\frac{\lambda}{4} \int_{\Omega} \frac{v_{\alpha}}{v_{\alpha}+\alpha} d x \\
& -\frac{\lambda}{1-\gamma} \int_{\Omega}\left[\left(v_{\alpha}+\alpha\right)^{1-\gamma}-\alpha^{1-\gamma}\right] d x \\
\geq & \frac{1}{4}\left\|v_{\alpha}\right\|^{2}+\frac{1}{12} \int_{\Omega} v_{\alpha}^{6} d x-\frac{\lambda}{1-\gamma} \int_{\Omega}\left(v_{\alpha}^{+}\right)^{1-\gamma} d x \\
\geq & \frac{1}{4}\left\|v_{\alpha}\right\|^{2}+\frac{1}{12} \int_{\Omega} v_{\alpha}^{6} d x-\frac{\lambda}{1-\gamma}|\Omega|^{\frac{5+\gamma}{6}} S^{-\frac{1-\gamma}{2}}\left\|v_{\alpha}\right\|^{1-\gamma}
\end{aligned}
$$

Obviously, $\left\{v_{\alpha}\right\}$ is bounded in $H_{0}^{1}(\Omega)$ for $0<\gamma<1$. Going if necessary to a subsequence, also denoted by $\left\{v_{\alpha}\right\}$, there exists $\left\{v_{*}\right\} \in H_{0}^{1}(\Omega)$ such that

$$
\left\{\begin{array}{l}
v_{\alpha} \rightarrow v_{*}, \quad \text { weakly in } H_{0}^{1}(\Omega), \\
v_{\alpha} \rightarrow v_{*}, \quad \text { strongly in } L^{p}(\Omega)(1 \leq p<6), \\
v_{\alpha}(x) \rightarrow v_{*}(x), \quad \text { a.e. in } \Omega .
\end{array}\right.
$$

Next, we prove that $\left(v_{*}, \phi_{u_{*}}\right)$ is a pair solution of system (1.1). Notice that $\left\{v_{\alpha}\right\}$ satisfies problem (2.3), with an easy computation, we get that

$$
-\Delta v_{\alpha} \geq v_{\alpha}^{5}+\frac{\lambda}{\left(v_{\alpha}+\alpha\right)^{\gamma}} \geq \min \left\{1, \frac{\lambda}{2^{\gamma}}\right\}
$$

it follows that $-\Delta v_{\alpha} \geq \min \left\{1, \frac{\lambda}{2^{\gamma}}\right\}$. We denote by $e$ the positive solution of

$$
\begin{cases}-\Delta u=1, & \text { in } \Omega \\ u=0, & \text { on } \partial \Omega\end{cases}
$$

Hence, we get that $e>0$ by using the strong maximum principle. For every $\Omega_{0} \subset \subset \Omega$, there exists $e_{0}>0$ such that $\left.e\right|_{\Omega_{0}} \geq e$; therefore, by comparison principle, we get

$$
v_{\alpha} \geq \min \left\{1, \frac{\lambda}{2^{\gamma}}\right\} e .
$$


In particular, from $\left.e\right|_{\Omega_{0}} \geq e>0$, we deduce that

$$
\left.v_{\alpha}\right|_{\Omega_{0}} \geq \min \left\{1, \frac{\lambda}{2^{\gamma}}\right\} e_{0}>0
$$

Now, we shall prove that $v_{\alpha} \rightarrow v_{*}$ as $\alpha \rightarrow 0$. It is similar to [29], for ang $\varphi \in H_{0}^{1}(\Omega)$, we have

$$
\int_{\Omega}\left(\nabla v_{*}, \nabla \varphi\right) d x-\int_{\Omega} \phi_{\nu_{*}} v_{*} \varphi d x-\int_{\Omega} v_{*}^{5} \varphi d x-\lambda \int_{\Omega} \nu_{*}^{-\gamma} \varphi d x=0
$$

Then, take a test function $\varphi=v_{*}$ in (4.3), there holds

$$
\left\|v_{*}\right\|^{2}-\int_{\Omega} \phi_{\nu_{*}} v_{*}^{2} d x-\int_{\Omega} v_{*}^{6} d x-\lambda \int_{\Omega} v_{*}^{1-\gamma} d x=0
$$

Without loss of generality, set $w_{\alpha}=v_{\alpha}-v_{*}$, then $\left\|w_{\alpha}\right\| \rightarrow 0$ as $\alpha \rightarrow 0$. Otherwise, there exists a subsequence (still denoted by $w_{\alpha}$ ) such that $\lim _{\alpha \rightarrow 0}\left\|w_{\alpha}\right\|=l>0$. Notice the given condition $\alpha>0$, we obtain $0 \leq \frac{v_{\alpha}}{\left(v_{\alpha}+\alpha\right)^{\gamma}} \leq v_{\alpha}^{1-\gamma}$, by the Hölder inequality and subadditivity, from (4.2), we have

$$
\begin{aligned}
\int_{\Omega} \frac{v_{\alpha}}{\left(v_{\alpha}+\alpha\right)^{\gamma}} d x & \leq \int_{\Omega} v_{\alpha}{ }^{1-\gamma} \leq \int_{\Omega}\left|w_{\alpha}\right|^{1-\gamma} d x+\int_{\Omega}{{ }^{*}}^{1-\gamma} d x \\
& \leq\left\|w_{\alpha}\right\|_{2}^{1-\gamma}|\Omega|^{\frac{1+\gamma}{2}}+\int_{\Omega} v_{*}{ }^{1-\gamma} d x \\
& \leq \int_{\Omega}{v_{*}}^{1-\gamma} d x+o(1) .
\end{aligned}
$$

Similarly,

$$
\int_{\Omega} v_{*}^{1-\gamma} d x \leq \int_{\Omega} \frac{v_{\alpha}}{\left(v_{\alpha}+\alpha\right)^{\gamma}} d x+o(1)
$$

Hence, one has

$$
\lim _{\alpha \rightarrow 0} \int_{\Omega} \frac{v_{\alpha}}{\left(v_{\alpha}+\alpha\right)^{\gamma}} d x=\int_{\Omega}{\nu_{*}}^{1-\gamma} d x
$$

Using the Brézis-Lieb lemma and by $\left\langle I_{\alpha}^{\prime}\left(v_{\alpha}\right), v_{\alpha}\right\rangle=0$, there holds

$$
\left\|w_{\alpha}\right\|^{2}+\left\|v_{*}\right\|^{2}-\int_{\Omega} \phi_{\nu_{*}}\left(v_{*}\right)^{2} d x-\int_{\Omega} w_{\alpha}^{6} d x-\int_{\Omega} v_{*}^{6} d x-\lambda \int_{\Omega} v_{*}^{1-\gamma} d x=o(1) .
$$

It follows from (4.4) and (4.5) that

$$
\left\|w_{\alpha}\right\|^{2}-\int_{\Omega} w_{\alpha}^{6} d x=o(1)
$$

Then (2.1) and (4.6) imply that

$$
l^{2} \geq S^{\frac{3}{2}}
$$


From (3.2), (4.4) and using the Young inequality, we have

$$
\begin{aligned}
I_{\lambda}\left(v_{*}\right) & =\frac{1}{2}\left\|\nu_{*}\right\|^{2}-\frac{1}{4} \int_{\Omega} \phi_{\nu_{*}} v_{0}^{2} d x-\frac{1}{6} \int_{\Omega} v_{*}^{6} d x-\frac{\lambda}{1-\gamma} \int_{\Omega} v_{*}^{1-\gamma} d x \\
& =\frac{1}{4}\left\|\nu_{*}\right\|^{2}+\frac{1}{12} \int_{\Omega} v_{*}^{6} d x-\lambda\left(\frac{1}{1-\gamma}-\frac{1}{4}\right) \int_{\Omega} v_{*}^{1-\gamma} d x \\
& \geq \frac{1}{4}\left\|\nu_{*}\right\|^{2}-\lambda\left(\frac{1}{1-\gamma}-\frac{1}{4}\right)|\Omega|^{\frac{5+\gamma}{6}} S^{-\frac{1-\gamma}{2}}\left\|v_{*}\right\|^{1-\gamma} \\
& \geq-D \lambda^{\frac{2}{1+\gamma}}
\end{aligned}
$$

where $D=\frac{1+\gamma}{4(1-\gamma)}\left(\frac{3+\gamma}{2}|\Omega|^{\frac{5+\gamma}{6}} S^{-\frac{1-\gamma}{2}}\right)^{\frac{2}{1+\gamma}}$. Moreover, from (3.15), (4.7), and the Brézis-Lieb lemma, one has

$$
\begin{aligned}
I_{\lambda}\left(v_{*}\right) & \leq I_{\lambda, \alpha}\left(v_{\alpha}\right)-\frac{1}{2}\left\|w_{\alpha}\right\|^{2}+\frac{1}{6} \int_{\Omega}\left|w_{\alpha}\right|^{6} d x \\
& <\frac{1}{3} S^{\frac{2}{2}}-D \lambda^{\frac{2}{1+\gamma}}-\frac{1}{3}\left\|w_{n}\right\|^{2} \\
& <\frac{1}{3} S^{\frac{2}{2}}-D \lambda^{\frac{2}{1+\gamma}}-\frac{1}{3} S^{\frac{3}{2}} \\
& =-D \lambda^{\frac{2}{1+\gamma}} .
\end{aligned}
$$

It is obvious that the above inequalities are impossibility. Thus, we get $l=0$, which yields $v_{\alpha} \rightarrow v_{*}$ in $H_{0}^{1}(\Omega)$ as $\alpha \rightarrow 0$.

In addition, we claim that $I_{\lambda, \alpha}$ is uniformly bounded. In fact, define a function $f(t)=$ $-(u+t)^{1-\gamma}+t^{1-\gamma}$, we easily get $f^{\prime}(t)<0$ for $t>0$. Obviously, $f(t)$ is decreasing for $0<t<1$. It follows that

$$
I_{\lambda, 1}(u)<I_{\lambda, \alpha}(u)<I_{\lambda, 0}(u)
$$

for $u \in H_{0}^{1}(\Omega)$. So the claim is true. Therefore, by (3.18), we have $I_{\alpha}\left(v_{*}\right)=\lim _{\alpha \rightarrow 0} I_{\lambda, \alpha} v_{\alpha}=$ $c>0$.

Similarly, by Theorem 3.7 , there exists $u_{*} \in H_{0}^{1}(\Omega)$ such that $u_{\alpha} \rightarrow u_{*}$ and $I_{\alpha}\left(u_{*}\right)=$ $\lim _{\alpha \rightarrow 0} I_{\lambda, \alpha}\left(u_{\alpha}\right)=d<0$.

Therefore, $u_{*}, v_{*}$ are two different positive solutions of problem (2.2). And $\left(u_{*}, \phi_{u_{*}}\right)$, $\left(v_{*}, \phi_{\nu_{*}}\right)$ are two pairs of different positive solutions of system (1.1). This completes the proof of Theorem 1.1.

\section{Acknowledgements}

The authors are grateful to the referees for carefully reading the paper and for their comments and suggestions.

Funding

This paper is supported by the National Natural Science Foundation of China (No. 11661021; No. 11861021), Innovation Group Major Program of Guizhou Province (No. KY[2016]029), Science and Technology Foundation of Guizhou Provincial Department of Education (No. KY[2019]1163).

Data sharing not applicable to this article as no data sets were generated or analyzed during the current study. 
Authors' contributions

All authors contributed equally and significantly in writing this article. All authors read and approved the final manuscript.

\section{Publisher's Note}

Springer Nature remains neutral with regard to jurisdictional claims in published maps and institutional affiliations.

\section{Received: 4 January 2020 Accepted: 3 March 2020 Published online: 06 March 2020}

\section{References}

1. Benci, V., Fortunato, D.: An eigenvalue problem for the Schrödinger-Maxwell equations. Topol. Methods Nonlinear Anal. 11, 283-293 (1998)

2. Ambrosetti, A., Ruiz, D.: Multiple bound states for the Schrödinger-Poisson problem. Commun. Contemp. Math. 10 391-404 (2008)

3. Ruiz, D.: The Schrödinger-Poisson equation under the effect of a nonlinear local term. J. Funct. Anal. 237, 655-674 (2006)

4. Papageorgiou, N.S., Radulescu, V.D., Repovs, D.D.: Positive solutions for nonlinear parametric singular Dirichlet problems. Bull. Math. Sci. 9(3), 1950011 (2019)

5. Papageorgiou, N.S., Radulescu, V.D., Repovs, D.D.: Nonlinear nonhomogeneous singular problems. Calc. Var. Partial Differ. Equ. 59(1), 9 (2020)

6. Rolando, S.: Multiple nonradial solutions for a nonlinear elliptic problem with singular and decaying radial potential. Adv. Nonlinear Anal. 8(1), 885-901 (2019)

7. Xue, Y.F., Tang, C.L.: Existence of a bound state solution for quasilinear Schrödinger equations. Adv. Nonlinear Anal. 8(1), 323-338 (2019)

8. Cerami, G., Vaira, G.: Positive solutions for some non-autonomous Schrödinger-Poisson systems. J. Differ. Equ. 248(3), 521-543 (2010)

9. D'Avenia, P., Pomponio, A., Vaira, G.: Infinitely many positive solutions for a Schrödinger-Poisson system. Nonlinear Anal. 74, 5705-5721 (2011)

10. Chen, J.: Multiple positive solutions of a class of non autonomous Schrödinger-Poisson systems. Nonlinear Anal., Real World Appl. 21, 13-26 (2015)

11. Sun, J., Wu, T., Feng, Z:: Multiplicity of positive solutions for a nonlinear Schrödinger-Poisson system. J. Differ. Equ. 260, 586-627 (2016)

12. Shen, Z.P., Han, Z.Q.: Multiple solutions for a class of Schrödinger-Poisson system with indefinite nonlinearity. J. Math. Anal. Appl. 426, 839-854 (2015)

13. He, X., Zou, W.: Existence and concentration of ground states for Schrödinger-Poisson equations with critical growth. J. Math. Phys. 53, 023702 (2012)

14. Chen, S., Tang, X.: Ground state solutions of Schrödinger-Poisson systems with variable potential and convolution nonlinearity. J. Math. Anal. Appl. 473(1), 87-111 (2019)

15. Liu, Z.L., Wang, Z.Q., Zhang, J.J.: Infinitely many sign-changing solutions for the nonlinear Schrödinger-Poisson system. Ann. Math. 195, 775-794 (2016)

16. Zhong, X.J., Tang, C.L.: Ground state sign-changing solutions for a Schrödinger-Poisson. Nonlinear Anal., Real World Appl. 39, 166-184 (2018)

17. Khoutir, S.: Infinitely many high energy radial solutions for a class of nonlinear Schrödinger-Poisson systems in $\mathbb{R}^{3}$. Appl. Math. Lett. 90, 139-145 (2019)

18. Azzollini, A., D’Avenia, P., Luisi, V.: Generalized Schrödinger-Poisson type systems. Commun. Pure Appl. Anal. 12(2), $1-12(2013)$

19. Alves, C.O., Souto, M.A.S.: Existence of least energy nodal solution for a Schrödinger-Poisson system in bounded domains. Z. Angew. Math. Phys. 65, 1153-1166 (2014)

20. Lei, C.Y., Liu, G.S.: Multiple positive solutions for a Schrödinger-Newton system with sign-changing potential. Comput. Math. Appl. 77, 631-640 (2019)

21. Fan, H.: Multiple positive solutions for Schrodinger-Poisson systems involving concave-convex nonlinearities. Electron. J. Differ. Equ. 2019, 86 (2019)

22. Zhang, Q.: Existence, uniqueness and multiplicity of positive solutions for Schrödinger-Poisson system with singularity. J. Math. Anal. Appl. 437, 160-180 (2016)

23. Lei, C.Y., Suo, H.M.: Positive solutions for a Schrödinger-Poisson system involving concave-convex nonlinearities. Comput. Math. Appl. 74, 1516-1524 (2017)

24. Zheng, W., Gan, W., Liu, S.: Existence of positive ground state solutions of Schrödinger-Poisson system involving negative nonlocal term and critical exponent on bounded domain. Bound. Value Probl. 2019(185), 1 (2019)

25. Brézis, H., Lieb, F.: A relation between pointwise convergence of functions and convergence of functionals. Proc. Am. Math. Soc. 88(3), 486-490 (1983)

26. Willem, M.: Minimax Theorems. Birkhäuser, Boston (1996)

27. Lei, C.Y., Liu, G.S., Guo, L.T.: Multiple positive solutions for a Kirchhoff type problem with a critical nonlinearity. Nonlinear Anal., Real World Appl. 31, 343-355 (2016)

28. Ambrosetti, A., Rabinowitz, P.H.: Dual variational methods in critical point theory and applications. J. Funct. Anal. 14, 349-381 (1973)

29. Lei, C.Y., Liao, J.F., Tang, C.L.: Multiple positive solutions for Kirchhoff type of problems with singularity and critical exponents. J. Math. Anal. Appl. 421, 521-538 (2015) 